\title{
DISPONIBILIDADE DE CÁLCIO EM LEITES INTEGRAL E DESNATADO ADICIONADOS DE FRUTAS, CAFÉ E ACHOCOLATADO
}

\begin{abstract}
O objetivo do presente estudo foi avaliar a disponibilidade do cálcio no leite de vaca (UHT) integral e desnatado misturados com: banana cultivar Nanica; mamão cultivar Formosa; maçã cultivar Gala com e sem casca; achocolatado em pó e infusão de café. As amostras foram submetidas às análises de composição centesimal, fatores antinutricionais (taninos, ácido oxálico e ácido fítico), minerais e disponibilidade de cálcio. Adotou-se delineamento experimental inteiramente ao acaso e os resultados obtidos foram submetidos à análise estatística, empregando-se o teste de Tukey e o teste de correlação de Pearson ao nível de 5\%. Quanto à composição centesimal, mineral e ao cálcio disponível, apenas os lipídios apresentaram diferença significativa entre as amostras compostas por leite integral e as compostas por leite desnatado. Somente o ferro apresentou correlação significativa positiva para a disponibilidade de cálcio. As amostras compostas por leite integral e desnatado, ambas misturadas com banana ou mamão apresentaram os melhores resultados para a disponibilidade de cálcio. As amostras formadas por misturas de leite integral e desnatado com achocolatado em pó apresentaram resultados numéricos superiores às demais amostras analisadas com relação ao teor de cálcio, cálcio dialisado e cálcio disponível em porção de $200 \mathrm{~mL} /$ amostra, provavelmente devido à formulação do achocolatado em pó. Levando-se em consideração os dados obtidos conclui-se que os melhores resultados foram apresentados pelos leites integral e desnatado com achocolatado e com banana. A maçã adicionada ao leite com ou sem casca não influenciou o resultado da disponibilidade do cálcio.
\end{abstract}

PALAVRAS-CHAVE: LEITE; FRUTAS; ACHOCOLATADO; CAFÉ; CÁLCIO - DISPONIBILIDADE.

* Mestranda, Programa de pós-graduação em Ciência e Tecnologia de Alimentos, Departamento de Agroindústria, Alimentos e Nutrição, Escola Superior de Agricultura "Luiz de Queiroz"/ Universidade de São Paulo (ESALQ/USP), Piracicaba, SP (e-mail: fagnogue@esalq.usp.br).

** Professora, doutora em Ciência dos Alimentos, Departamento de Agroindústria, Alimentos e Nutrição, ESALQ/USP, Piracicaba, SP (e-mail: sgcbraza@esalq.usp.br). 


\section{INTRODUÇÃO}

As mudanças demográficas observadas nas regiões mais desenvolvidas do mundo vêm adquirindo grande interesse público devido a óbitos e enfermidades relacionadas com as doenças crônico-degenerativas, entre as quais a osteoporose (FRAZÃO e NAVEIRA, 2006). Estima-se que a porcentagem de doenças crônicas não-transmissíveis aumente em $57 \%$ até o ano de 2020 , incluindo os países em desenvolvimento (OMS, 2003).

A incidência de osteoporose aumenta com a longevidade da população (ERVITI, 2003; GUIMARÃES, BRAUM e GOMEZ, 2005), especialmente em mulheres a partir do período pós-menopausa (ERVITI, 2003; ÁNGEL et al., 2003). Segundo KOWALSKI, SJENZFELD e FERRAZ (2001), essa enfermidade óssea afeta cerca de $30 \%$ das mulheres nesse período.

Considera-se a boa formação óssea um dos meios mais eficazes de prevenção da osteoporose em idades avançadas (MATKOVIC, 1992). Neste sentido, SILVA, TEIXEIRA e GOLDBERG (2004), LERNER et al. (2000) e McCLUNG (2003) preconizam a necessidade de suprimento diário mínimo constante de cálcio durante a fase de crescimento humano. No entanto, com relação aos hábitos alimentares de crianças e adolescentes, vários estudos apontam como insatisfatório o consumo de cálcio de acordo com o preconizado para as respectivas faixas etárias. Mesmo assim, o leite figura entre os alimentos mais consumidos e citados em pesquisas de consumo alimentar (FARIAS JÚNIOR e OSÓRIO, 2005; LERNER et al., 2000; MAESTRO, 2003; SANTOS et al., 2005; CARMO et al., 2006).

Para adequar nutricionalmente a dieta, com o objetivo de atingir o máximo de massa óssea de cada indivíduo, avaliar somente a quantidade de cálcio consumido pela alimentação não é suficiente (CRAWFORD et al., 2002). Vários fatores interferem na biodisponibilidade do cálcio, fisiológica e nutricionalmente. Entre os fatores fisiológicos tem-se a presença de vitamina $D$; níveis de cálcio e fósforo, idade do indivíduo e gravidez. Entre os fatores dietéticos tem-se a presença de lactose, proteínas, cálcio, ácido fítico e balanço fósforo/cálcio (GUÉGUEN e POINTLLART, 2000).

Considerando o consumo de café (FARIAS, JUNIOR e OSÓRIO, 2005); a crescente ingestão de achocolatado em pó (AQUINO e PHILLIPI, 2002); a expressiva participação da banana, entre as frutas, na dieta alimentar de crianças e adolescentes (FARIAS JUNIOR e OSÓRIO, 2005) e, a expansão da produção de maçãs (EPAGRI, 2002; KOVALESKI, 2004; MELLO e BORGES JÚNIOR, 2004), além da grande produção brasileira de mamão (FAO, 2004) há necessidade de verificar as alterações na disponibilidade do cálcio do leite com a adição desses alimentos.

Este trabalho teve como objetivo avaliar a disponibilidade do cálcio presente no leite de vaca (UHT) integral e desnatado, ambos misturados com banana, maçã, mamão, achocolatado em pó e com infusão de café.

\section{MATERIAL E MÉTODOS}

As matérias-primas utilizadas para esta pesquisa foram adquiridas no comércio da cidade de Piracicaba, São Paulo: 12 litros de leite de vaca integral (UHT); 12 litros de leite de vaca desnatado (UHT); aproximadamente $480 \mathrm{~g}$ de mamão cultivar formosa; $455 \mathrm{~g}$ de banana cultivar nanica; $480 \mathrm{~g}$ de maçã cultivar gala; $425,25 \mathrm{~g}$ de achocolatado em pó solúvel; $50 \mathrm{~g}$ café torrado e moído e $60 \mathrm{~g}$ de açúcar refinado.

A partir das matérias-primas foram obtidas 2 amostras de leite e 12 amostras de misturas, nas seguintes proporções: Amostra 1 = leite integral (UHT); Amostra $2=$ leite desnatado (UHT); Amostra $3=$ leite integral (UHT) e banana nanica (5:0,9); Amostra 4 = leite desnatado (UHT) e banana nanica (5:0,9); Amostra 5 = leite integral (UHT) e mamão formosa (5:1); Amostra $6=$ leite desnatado (UHT) e mamão formosa (5:1); Amostra 7 = leite integral (UHT) e maçã gala sem casca (5:1); Amostra 8 = leite desnatado (UHT) e maçã gala sem casca (5:1); Amostra 9 = leite integral (UHT) e maçã gala com casca (5:1); Amostra 10 = leite desnatado (UHT) e maçã gala com casca (5:1); Amostra 11 = leite integral (UHT) e achocolatado em pó (8:1); Amostra 12 = leite desnatado (UHT) e achocolatado em pó (8:1); Amostra 13 = leite integral (UHT) e café (infusão) (3:1); Amostra 14 = leite desnatado (UHT) e café (infusão) (3:1). 


\subsection{COMPOSIÇÃO CENTESIMAL}

As análises químicas da matéria seca, cinzas e proteínas das 14 amostras foram realizadas de acordo com a metodologia descrita pela AOAC (2006). O teor de lipídios foi analisado segundo metodologia de BLIGH e DYER (1959).

\subsection{FATORESANTINUTRICIONAIS}

Os taninos foram analisados segundo a metodologia descrita por PRICE et al. (1980), o ácido oxálico pelo método de MOIR (1953) e o teor de ácido fítico das amostras conforme o método proposto por GRYNSPAN e CHERYAN (1989).

\subsection{MINERAIS}

A determinação dos minerais seguiu o método descrito por SARRUGE e HAAG (1974), efetuandose digestão nitro-perclórica com posterior leitura em diferentes comprimentos de onda.

\subsection{DIÁLISE DE CÁLCIO}

A análise da diálise do cálcio foi realizada segundo o método proposto por ÜNAL, NEHIR e KILIÇ (2005), o qual consiste na simulação da digestão gastrointestinal com solução de pepsina-HCI e solução de pancreatina-sais de bile. Inicialmente o pH das amostras foi ajustado para 2,0 com HCl 6,0 M. Em seguida, as amostras foram incubadas em banho-maria com agitação durante duas horas a $37^{\circ} \mathrm{C}$, sendo o $\mathrm{pH}$ verificado a cada trinta minutos e ajustado para 2,0 quando necessário. Mediu-se a acidez titulável e adicionou-se solução pancreatina-bile. A diálise foi realizada durante duas horas com sacos de diálise contendo $\mathrm{NaHCO}_{3} 1 \mathrm{M}$, equivalente a acidez titulável. O conteúdo do cálcio foi analisado por espectrofotometria de absorção atômica e a disponibilidade do cálcio calculada pela seguinte equação:

\%Ca disponível $=[$ Ca dialisado $(\mathrm{mg} / 100 \mathrm{~mL}) /$ Ca da amostra $(\mathrm{mg} / 100 \mathrm{~mL})] \times 100$

\subsection{ANÁLISE ESTATÍSTICA}

Todas as análises foram realizadas em triplicata, com delineamento inteiramente ao acaso. Foram empregados o teste $\mathrm{F}$ e o teste de Tukey para diferença de média, considerando nível de significância de $5 \%$. Essas análises foram realizadas usando-se o programa estatístico SAS (1996). Para estabelecer se houve correlação entre a disponibilidade de cálcio, composição centesimal, antinutricionais e minerais foi utilizado o teste de correlação de Pearson (MICROSOFT, 2003).

\section{RESULTADOS E DISCUSSÃO}

A composição centesimal das amostras foi determinada com o objetivo de caracterizar os componentes utilizados na pesquisa. Os resultados obtidos nas análises estão apresentados na Tabela 1 e os resultados da comparação entre os leites integral e desnatado na Tabela 2. Somente o componente lipídio apresentou diferença significativa $(p \leq 0,05)$, quando se compara as amostras compostas por leite integral e desnatado (Tabela 2).

A diferença no teor de umidade encontrada entre as amostras pesquisadas apresentou variação entre $91,12 \%$ (amostra de leite desnatado com mamão) e 79,16\% (leite integral com achocolatado) (Tabela 1). 


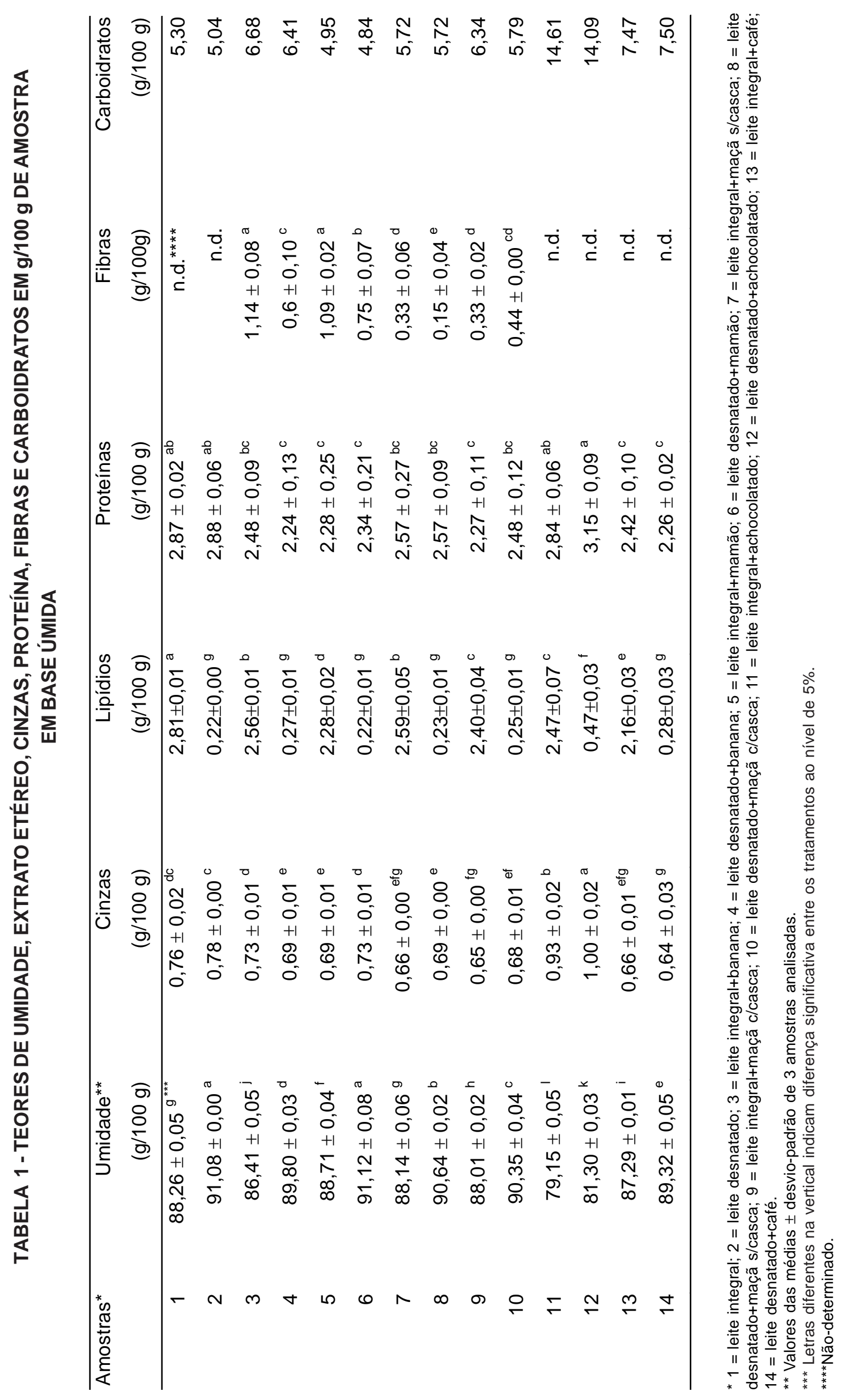


As amostras formadas por misturas com achocolatado apresentaram teores superiores para cinzas quando comparadas às demais e aos valores citados na literatura (UNICAMP, 2006; ITO, 2003; USP, 2004; PHILIPPI, 2001; ABPM, 2006). O achocolatado em pó apresenta a maior taxa de concentração para cinzas dentre todos os alimentos utilizados neste estudo, quando considerado separadamente, de acordo com as Tabelas apresentadas pela UNICAMP (2006) e por ITO (2003).

O resultado obtido nesta pesquisa (Tabela 1$)$ para o leite de vaca integral $(2,81 \%)$ mostrou-se inferior aos teores descritos por FRANCO (2001), ITO (2003), PHILIPPI (2001) e ÜNAL, NEHÍR e KILIÇ (2005). O leite integral UHT deve apresentar o mínimo de 3\% de lipídios, conforme a Resolução 2 de 19 de novembro de 2002 (BRASIL, 2002), sendo aceitáveis variações de $\pm 20 \%$ para todos os nutrientes descritos nos rótulos das embalagens de acordo com a RDC 360, de 23 de dezembro de 2003 (BRASIL, 2003). Quanto ao leite desnatado UHT, o teor de lipídios foi de $0,22 \%$ (Tabela 1 ) que se enquadrou no intervalo de 0,10\% a 0,36\% indicado por FRANCO, 2001; ITO, 2003; PHILIPPI, 2001; ÜNAL, NEHIR e KILIÇ, 2005.

Entre as amostras pesquisadas compostas por misturas, as formadas por achocolatado revelaram os maiores índices protéicos (Tabela 1). O achocolatado em pó apresenta o maior teor protéico quando considerado cada alimento separadamente, o que pode justificar o resultado obtido para as amostras 11 e 12 (UNICAMP, 2006; ITO, 2003; USP, 2004; PHILIPPI, 2001; ABPM, 2006). A proporção adicionada ao leite de cada alimento provavelmente também influenciou o resultado.

Para os carboidratos, os maiores valores numéricos encontrados foram $14,61 \%$ e $14,09 \%$ para as amostras compostas de leite integral com achocolatado e leite desnatado com achocolatado, respectivamente (Tabela 1). Tal resultado deve-se à composição do achocolatado em pó, o qual apresenta o maior teor para carboidratos dentre todos os alimentos utilizados nesta pesquisa para compor as amostras (UNICAMP, 2006; ITO, 2003).

\section{TABELA 2 - COMPARAÇÃO DAS AMOSTRAS COMPOSTAS POR LEITE INTEGRALE DESNATADO}

\begin{tabular}{lll}
\hline Variável & $\begin{array}{l}\text { Média das amostras } \\
\text { com leite integral }\end{array}$ & $\begin{array}{l}\text { Média das amostras } \\
\text { com leite desnatado }\end{array}$ \\
\hline Umidade & $86,569^{a^{x}}$ & $89,087^{\mathrm{a}}$ \\
Cinza & $0,726^{\mathrm{a}}$ & $0,744^{\mathrm{a}}$ \\
Lipídios & $2,467^{\mathrm{a}}$ & $0,277^{\mathrm{b}}$ \\
Proteínas & $2,533^{\mathrm{a}}$ & $2,560^{\mathrm{a}}$ \\
Fibras & $0,723^{\mathrm{a}}$ & $0,485^{\mathrm{a}}$ \\
Carboidratos & $7,296^{\mathrm{a}}$ & $7,056^{\mathrm{a}}$ \\
\hline
\end{tabular}

* Letras diferentes na horizontal indicam diferença significativa entre as amostras em nível de 5\%.

Os resultados encontrados para antinutricionais foram baixos para todos os componentes analisados (Tabela 3), indicando que essas substâncias não são relevantes nas misturas com o leite.

As taxas de taninos encontradas nas amostras investigadas variaram entre $0,01 \% \mathrm{mEqcatequina}$ (amostras 8; 9 e 10) e 0,09\% mEqcatequina (amostra 12) que são consideradas quantidades baixas (Tabela 3). Para a maçã da variedade Gala, CURTI (2003) obteve valor de 0,16 g/100 g para taninos.

Somente foram analisadas as amostras 11 e 12 quanto ao ácido oxálico, pois são as únicas que podem apresentar quantidade mensurável desse componente. $O$ teor de ácido oxálico para a amostra composta por leite integral e achocolatado em pó foi $0,30 \%$ e para a amostra composta por leite desnatado e achocolatado 0,22\% (Tabela 3). Os níveis de ácido oxálico presentes nos alimentos podem variar conforme a época do ano e tipo de cultivo (BOAVENTURA, 1998).

Os teores de ácido fítico das amostras pesquisadas variaram entre 0,001 e 0,003 mg/g. Apenas as amostras formadas por misturas com achocolatado apresentaram taxas de 0,003 mg/g de ácido fítico. Os demais resultados variaram entre $0,001 \mathrm{mg} / \mathrm{g}$ e $0,002 \mathrm{mg} / \mathrm{g}$ (Tabela 3). Esses teores são baixos quando comparados aos de grãos avaliados por HEANEY, WEAVER e FITZSIMMONS (1991). 
TABELA 3 - TEORES DOS FATORES ANTINUTRICIONAIS DAS AMOSTRAS ANALISADAS

\begin{tabular}{|c|c|c|c|}
\hline Amostras ${ }^{*}$ & $\begin{array}{l}\text { Taninos }{ }^{*} \\
\text { (\%mEqcatequina) }\end{array}$ & $\begin{array}{l}\text { Ácido oxálico }{ }^{*} \\
\text { (\%) }\end{array}$ & $\begin{array}{l}\text { Ácido fitico\#" } \\
\text { (mg/g) }\end{array}$ \\
\hline 1 & n.d. & n.d. & n.d. \\
\hline 2 & n.d. & n.d. & n.d. \\
\hline 3 & $0,03 \pm 0,00^{e^{\cdots}}$ & n.d. & $0,002 \pm 0,00^{b}$ \\
\hline 4 & $0,03 \pm 0,00^{e}$ & n.d. & $0,001 \pm 0,00^{\circ}$ \\
\hline 5 & $0,06 \pm 0,00^{\circ}$ & n.d. & $0,002 \pm 0,00^{b}$ \\
\hline 6 & $0,04 \pm 0,00^{d}$ & n.d. & $0,002 \pm 0,00^{b}$ \\
\hline 7 & $0,02 \pm 0,00^{\top}$ & n.d. & $0,002 \pm 0,00^{b}$ \\
\hline 8 & $0,01 \pm 0,00^{9}$ & n.d. & $0,001 \pm 0,00^{c}$ \\
\hline 9 & $0,01 \pm 0,00^{9}$ & n.d. & $0,002 \pm 0,00^{b}$ \\
\hline 10 & $0,01 \pm 0,00^{9}$ & n.d. & $0,001 \pm 0,00^{\circ}$ \\
\hline 11 & $0,03 \pm 0,00^{e}$ & $0,30 \pm 0,05^{a}$ & $0,003 \pm 0,00^{a}$ \\
\hline 12 & $0,09 \pm 0,00^{a}$ & $0,22 \pm 0,04^{\mathrm{a}}$ & $0,003 \pm 0,00^{a}$ \\
\hline 13 & $0,05 \pm 0,00^{\circ}$ & n.d. & $0,001 \pm 0,00^{\circ}$ \\
\hline 14 & $0,05 \pm 0,00^{\circ}$ & n.d. & $0,001 \pm 0,00^{\circ}$ \\
\hline
\end{tabular}

* 1 = leite integral; 2 = leite desnatado; 3 = leite integral+banana; 4 = leite desnatado+banana; 5 = leite integral+mamão; 6 = leite desnatado+mamão; 7 = leite integral+maçã s/casca; 8 = leite desnatado+maçã s/casca; 9 = leite integral+maçã cl casca; 10 = leite desnatado+maçã c/casca; 11 = leite integral+achocolatado; 12 = leite desnatado+achocolatado; 13 = leite integral+café; 14 = leite desnatado+café.

** Valores das médias \pm desvio-padrão de 3 amostras analisadas.

*** Letras diferentes na vertical indicam diferença significativa entre os tratamentos ao nível de $5 \%$.

**** Não-determinado.

Os resultados encontrados para minerais não apresentaram diferença significativa $(p>0,05)$ entre as amostras compostas por leite integral e por leite desnatado (Tabela 4).

\section{TABELA 4 - COMPARAÇÃO DAS AMOSTRAS COMPOSTAS POR LEITE} INTEGRALE DESNATADO

\begin{tabular}{lll}
\hline Variável & $\begin{array}{l}\text { Média das amostras } \\
\text { com leite integral }\end{array}$ & $\begin{array}{l}\text { Média das amostras } \\
\text { com leite desnatado }\end{array}$ \\
\hline Cobre & $0,043^{\text {a }}$ & $0,031^{\text {a }}$ \\
Ferro & $0,826^{\text {a }}$ & $0,591^{\text {a }}$ \\
Manganês & $0,030^{\text {a }}$ & $0,051^{\text {a }}$ \\
Zinco & $0,410^{\text {a }}$ & $0,410^{\text {a }}$ \\
Fósforo & $89,346^{\text {a }}$ & $83,683^{\text {a }}$ \\
Potássio & $148,380^{\text {a }}$ & $143,810^{\text {a }}$ \\
Magnésio & $11,724^{\text {a }}$ & $10,276^{\text {a }}$ \\
Erxofre & $28,714^{\text {a }}$ & $30,913^{\text {a }}$ \\
Sódio & $51,476^{\text {a }}$ & $50,879^{\text {a }}$ \\
Cálcio & $125,030^{\text {a }}$ & $123,660^{\text {a }}$ \\
\hline
\end{tabular}

\footnotetext{
* Letras diferentes na horizontal indicam diferença significativa entre as amostras em nível de 5\%.
}

As amostras compostas por leite integral e desnatado com achocolatado em pó (amostras 11 e 12) apresentaram valores numéricos superiores, quando comparadas às demais para as taxas de cálcio, magnésio, manganês, cobre e ferro. Tal diferença deve-se, provavelmente, à composição do achocolatado (Tabela 5 e Tabela 6). 

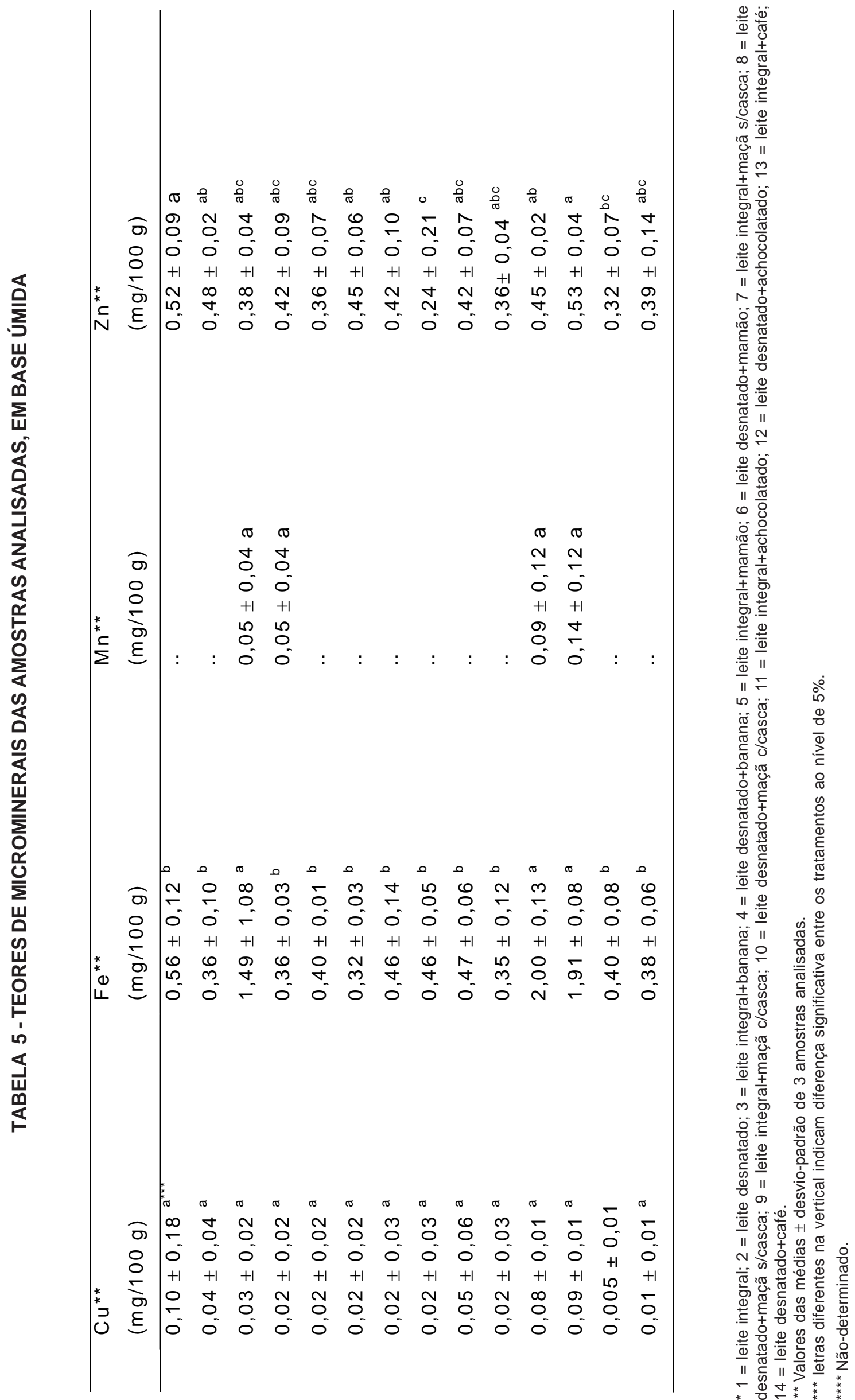


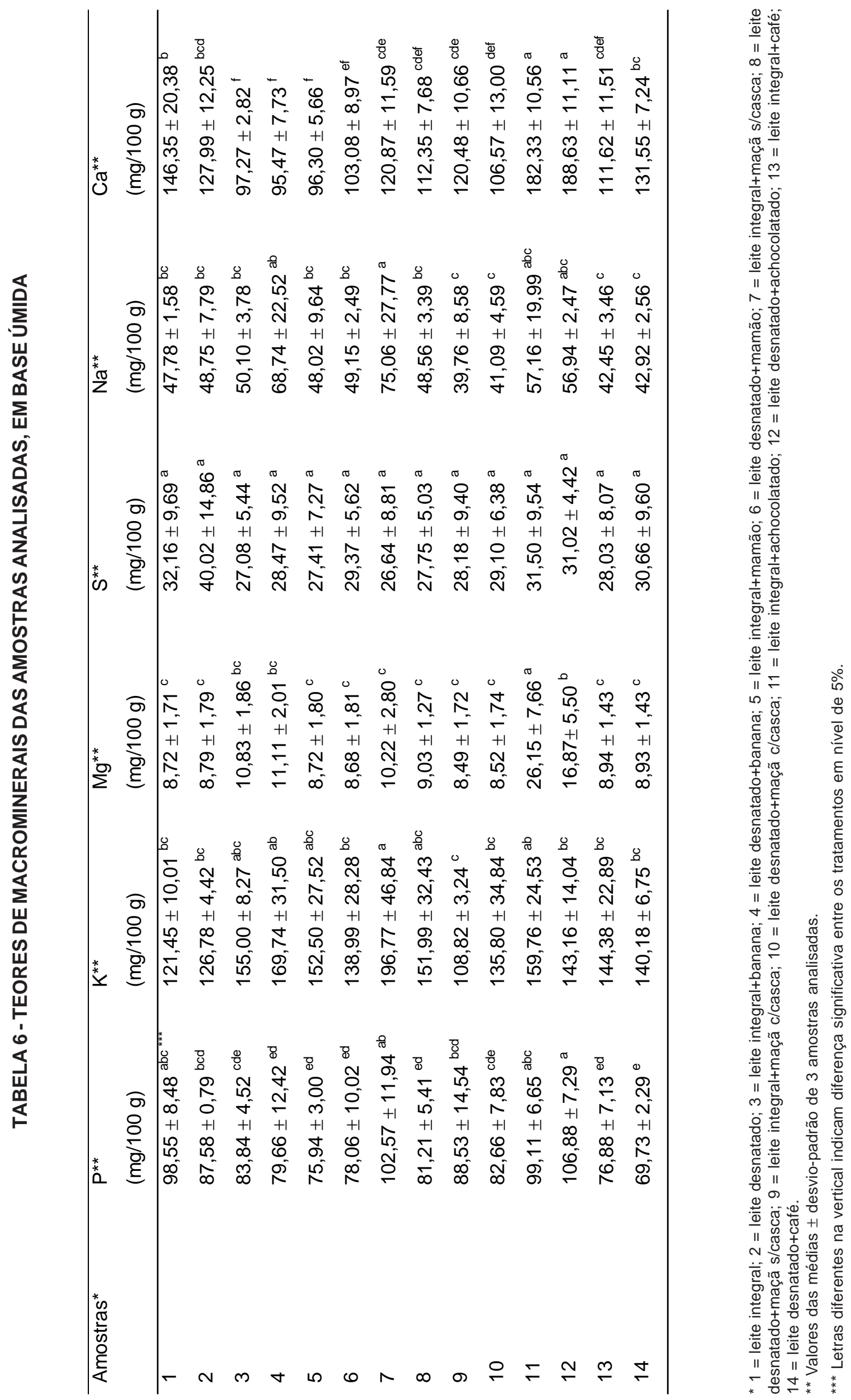


Entre os fatores que interferem nos teores de minerais dos vegetais pode-se citar: a variedade, o estágio de maturação, condições e características do solo, fertilização, irrigação e condições climáticas (TAHVONEN, 1993).

ÜNAL, NEHÍR e KILIÇ (2005), estudando a disponibilidade de cálcio em leite, derivados de leite e fórmulas infantis encontraram valores médios de 108,50 mg/100 g para o leite integral e $114,00 \mathrm{mg} / 100 \mathrm{~g}$ para o leite desnatado, ambos inferiores aos obtidos nesta pesquisa.

Pode-se observar que houve diferença nos resultados referentes ao cálcio dialisado (Tabela 7). A maior quantidade obtida foi de $41,04 \mathrm{mg} / 100 \mathrm{~mL}$ de cálcio dialisado para a amostra 12 (composta por leite desnatado misturado com achocolatado), e as menores de $21,71 \mathrm{mg} / 100 \mathrm{~mL}$ para a amostra 13 (composta por leite integral com café) e $21,72 \mathrm{mg} / 100 \mathrm{~mL}$ para a amostra 9 (composta por leite integral e maçã com casca).

A quantidade de cálcio dialisado obtida neste estudo para a amostra de leite integral $(28,31 \mathrm{mg} / 100 \mathrm{~mL})$ e para a amostra de leite desnatado $(32,39 \mathrm{mg} / 100 \mathrm{~mL})$ mostrou-se semelhante aos resultados da pesquisa realizada por ÜNAL, NEHÍR e KILIÇ (2005), que encontraram 28,10 mg/ $100 \mathrm{~mL}$ e $32,17 \mathrm{mg} / 100 \mathrm{~mL}$ para o leite integral e desnatado, respectivamente. Ambos os valores encontrados nesta pesquisa para o cálcio dialisado (Tabela 7) foram superiores ao descrito por ROIG et al. (1999a) para o leite de vaca $(19,80 \mathrm{mg} / 100 \mathrm{~mL})$.

As quantidades de cálcio dialisado nas amostras compostas por misturas de leite com maçã foram numericamente maiores nas amostras 7 (24,68 mg/100 g) e $8(25,72 \mathrm{mg} / 100 \mathrm{~g})$ formadas por maçã sem casca (Tabela 7) em relação às com casca, amostras 9 (21,72 mg/100 g) e $10(23,20 \mathrm{mg} /$ 100 g). Segundo BOSSCHER, VAN CAILLIE-BERTRAND e DEELSTRA (2001), as fibras são classificadas como potentes inibidores da absorção de minerais e elementos traços em geral, fato que possivelmente explica os resultados descritos acima.

Compara-se na Tabela 8, a disponibilidade de cálcio entre as amostras compostas por leite integral e compostas por leite desnatado.

\section{TABELA 7 - QUANTIDADE DE CÁLCIO DIALISADO E PORCENTAGEM DA DISPONIBILIDADE DE CÁLCIO}

\begin{tabular}{|c|c|c|}
\hline Amostras & $\begin{array}{l}\text { Quantidade de cálcio dialisado } \\
\text { (mg/100 mL) * }\end{array}$ & Disponibilidade do cálc io $(\%)$ * \\
\hline Leite integral & $28,31 \pm 3,62^{c}$ & $19,84 \pm 2,53^{\text {काT }}$ \\
\hline Leite desnatado & $32,39 \pm 1,73^{b}$ & $19,15 \pm 3,42^{\top}$ \\
\hline Leite integral+banana & $23,98 \pm 1,68^{\mathrm{d}}$ & $24,82 \pm 1,74$ \\
\hline Leite desnatado+banana & $27,14 \pm 1,24^{\mathrm{c}}$ & $28,41 \pm 1,43^{a}$ \\
\hline Leite integral+mamẫo & $25,77 \pm 2,59^{d x}$ & $27,70 \pm 2,78^{a}$ \\
\hline Leite desnatado+mamấo & $23,93 \pm 1,45^{c k}$ & $24,61 \pm 1,49^{\mathrm{bc}}$ \\
\hline Leite integral+maçẫ s'casca & $24,68 \pm 2,04^{d b e}$ & $19,51 \pm 1,82^{\mathrm{er}}$ \\
\hline Leite desnatado+maçẫ s' casca & $25,72 \pm 3,04^{\mathrm{c} e}$ & $23,29 \pm 2,74 \propto$ \\
\hline Leite integral+maçẫ cl casca & $21,72 \pm 2,29^{e}$ & $19,23 \pm 2,03^{\top}$ \\
\hline Leite desnatado+maçẫ cl casca & $23,20 \pm 1,04$ & $23,03 \pm 1,05^{\alpha k}$ \\
\hline Leite integral+achocolatado & $37,53 \pm 2,08^{a}$ & $20,79 \pm 1,15$ \\
\hline Leite desnatado+achocolatado & $41,04 \pm 2,60^{a}$ & $22,21 \pm 1,41$ \\
\hline Leite integral+café & $21,71 \pm 0,96^{\mathrm{e}}$ & $19,84 \pm 0,88^{\text {der }}$ \\
\hline Leite desnatado+café & $24,19 \pm 5,26$ & $18,62 \pm 4,05^{\top}$ \\
\hline
\end{tabular}

* 1 = leite integral; 2 = leite desnatado; 3 = leite integral+banana; 4 = leite desnatado+banana; 5 = leite integral+mamão; 6 = leite desnatado+mamão; 7 = leite integral+maçã s/casca; 8 = leite desnatado+maçã s/casca; 9 = leite integral+maçã c/ casca; 10 = leite desnatado+maçã c/casca; 11 = leite integral+achocolatado; 12 = leite desnatado+achocolatado; $13=$ leite integral+café; 14 = leite desnatado+café. 
TABELA 8 - COMPARAÇÃO DAS AMOSTRAS COMPOSTAS POR LEITE INTEGRALE DESNATADO

\begin{tabular}{lll}
\hline Variável & $\begin{array}{l}\text { Média das amostras } \\
\text { com leite integral }\end{array}$ & $\begin{array}{l}\text { Média das amostras } \\
\text { com leite de snatado }\end{array}$ \\
\hline Disponibilidade de cálcio & $21,676^{{ }^{\star x}}$ & $22,760^{a}$ \\
\hline
\end{tabular}

*Letras diferentes na horizontal indicam diferença significativa entre as amostras ao nível de 5\%.

Os resultados para a porcentagem de disponibilidade de cálcio não apresentaram correlação com a composição centesimal, assim como não demonstraram correlação com os fatores antinutricionais (Tabela 9). Entretanto, KENNEFICK e CASHMAN (2000) relataram influência negativa de fitatos e oxalatos em relação à disponibilidade de cálcio em refeições semi-sintéticas. A ausência de influência desses fatores nas amostras pode ser decorrente do baixo teor ou inexistência dos mesmos.

\section{TABELA 9 - TESTE DE CORRELAÇÃO PARA A PORCENTAGEM DE DISPONIBILIDADE DE CÁLCIO, COMPOSIÇÃO CENTESIMAL E ANTINUTRICIONAIS NAS AMOSTRAS PESQUISADAS}

\begin{tabular}{lll}
\hline Variável & F significaçã̃o & R quadrado ajustado \\
\hline Umidade & $0,6084^{\mathrm{ns}}$ & $-0,0589$ \\
Cinza & $0,9127^{\mathrm{ns}}$ & $-0,0822$ \\
Lipídios & $0,5019^{\mathrm{ns}}$ & $-0,0417$ \\
Proteínas & $0,2201^{\mathrm{ns}}$ & 0,0493 \\
Fibras & $0,0975^{\mathrm{ns}}$ & 0,2891 \\
Carboidratos & $0,5764^{\mathrm{ns}}$ & $-0,0544$ \\
Taninos & $0,7573^{\mathrm{ns}}$ & $-0,0890$ \\
Ac.fítico & $0,8039^{\mathrm{ns}}$ & $-0,0929$ \\
Ac.oxálico & $0,1106^{\mathrm{ns}}$ & 0,3882 \\
\hline
\end{tabular}

Ns = correlação não-significativa entre os tratamentos ao nível de 5\%.

Entre os minerais, apenas o ferro apresentou correlação positiva com a disponibilidade de cálcio (Tabela 10). De maneira geral observou-se diferença nos resultados obtidos referente à disponibilidade de cálcio. A maior porcentagem obtida $(28,41 \%)$ foi para a amostra 4 (composta por leite desnatado misturado com banana) e a menor (18,62\%) para a amostra 14 (composta por leite desnatado com café). Provavelmente isso se deve à composição da banana que apresenta compostos derivados de aminoácidos na forma livre, o que poderia servir como complexador do cálcio, formando quelatos.

A taxa para disponibilidade de cálcio obtida neste estudo para a amostra de leite integral $(19,84 \%)$ e de leite desnatado $(19,15 \%)$ mostrou-se inferior que a constatada por ÜNAL, NEHÍR e 
KILIÇ (2005), que encontraram valores de $25,87 \%$ e $28,19 \%$ para o leite integral e desnatado, respectivamente. O resultado encontrado para o leite integral (Tabela 7) foi semelhante ao descrito por ROIG et al. (1999b), cuja taxa apresentada para a disponibilidade de cálcio presente no leite de vaca foi de 20,0\%. Enquanto os valores para a disponibilidade de cálcio descritos por BOSSCHER et al. (1998) foram 27,7\%, 31,5\% e 21,9\% para o leite de pré-escolares, leite semi-desnatado e fórmulas infantis, respectivamente.

\section{TABELA 10 - TESTE DE CORRELAÇÃO PARA A PORCENTAGEM DE DISPONIBILIDADE DE CÁLCIO E QUANTIDADE DE MINERAIS NAS AMOSTRAS PESQUISADAS}

\begin{tabular}{lll}
\hline Variável & F significação & R quadrado ajustado \\
\hline $\mathrm{P}$ & $0,2566^{\mathrm{ns}}$ & 0,0312 \\
$\mathrm{Ca}$ & $0,0793^{\mathrm{ns}}$ & 0,1708 \\
$\mathrm{~K}$ & $0,2737^{\mathrm{ns}}$ & 0,0237 \\
$\mathrm{Na}$ & $0,4238^{\mathrm{ns}}$ & $-0,0248$ \\
$\mathrm{Mg}$ & $0,8752^{\mathrm{ns}}$ & $-0,0810$ \\
$\mathrm{~S}$ & $0,1554^{\mathrm{ns}}$ & 0,0908 \\
$\mathrm{Mn}$ & $0,1451^{\mathrm{ns}}$ & 0,2477 \\
$\mathrm{Fe}$ & $0,0334^{\mathrm{s}}$ & 0,5547 \\
$\mathrm{Cu}$ & $0,0752^{\mathrm{ns}}$ & 0,4012 \\
$\mathrm{Zn}$ & $0,5618^{\mathrm{ns}}$ & $-0,1141$ \\
\hline
\end{tabular}

Ns = correlação não-significativa entre os tratamentos ao nível de 5\%. $\mathrm{S}=$ correlação significativa entre os tratamentos ao nível de $5 \%$.

Os resultados deste estudo não apresentaram correlação entre os níveis de cálcio disponível e lipídios como demonstra a Tabela 9. Pesquisas conduzidas por ÜNAL, NEHIR e KILIÇ (2005) e GUÉGUEN e POINTILLART (2000), também não constataram influência negativa do extrato etéreo sobre a disponibilidade de cálcio.

Observou-se que as misturas de leite (integral e desnatado) com banana e com mamão apresentaram as maiores taxas para disponibilidade de cálcio (Tabela 7). BOSSCHER et al. (2002) pesquisaram a disponibilidade de cálcio, ferro e zinco em fórmulas infantis. Observaram as seguintes correlações após aplicação do teste de Pearson: correlação positiva entre proteína e cálcio, lipídios e cálcio, cálcio e ferro e; correlação negativa entre fibra dietética e cálcio. Já os resultados das correlações entre os componentes das fórmulas e a disponibilidade de cálcio foram: positiva para carboidratos e negativa para proteínas, cálcio, fósforo, magnésio e ferro não-heme.

Com relação às amostras compostas por misturas de leite com maçã, as taxas foram bastante semelhantes quando comparadas com os resultados das amostras formadas por leite integral com maçã com casca $(19,23 \%)$ e leite integral com maçã sem casca $(19,51 \%)$. Também foram semelhante entre as misturas de leite desnatado com maçã sem casca $(23,29 \%)$ e de leite desnatado e maçã com casca $(23,03 \%)$. 
Para a quantidade de cálcio disponível em $200 \mathrm{~mL}$ de cada amostra pesquisada nota-se que as amostras 11 e 12 (leite integral com achocolatado em pó e leite desnatado com achocolatado em pó, respectivamente) mostraram os melhores resultados. Tal fato se deve provavelmente à formulação do produto achocolatado em pó pois, ainda que em termos de cálcio dialisado não tenham evidenciando os melhores resultados, apresentaram quantidades de cálcio bastante superiores às demais amostras analisadas (Figura 1).

\section{FIGURA 1 - QUANTIDADE DE CÁLCIO DISPONÍVEL EM PORÇÃO DE 200 ML}

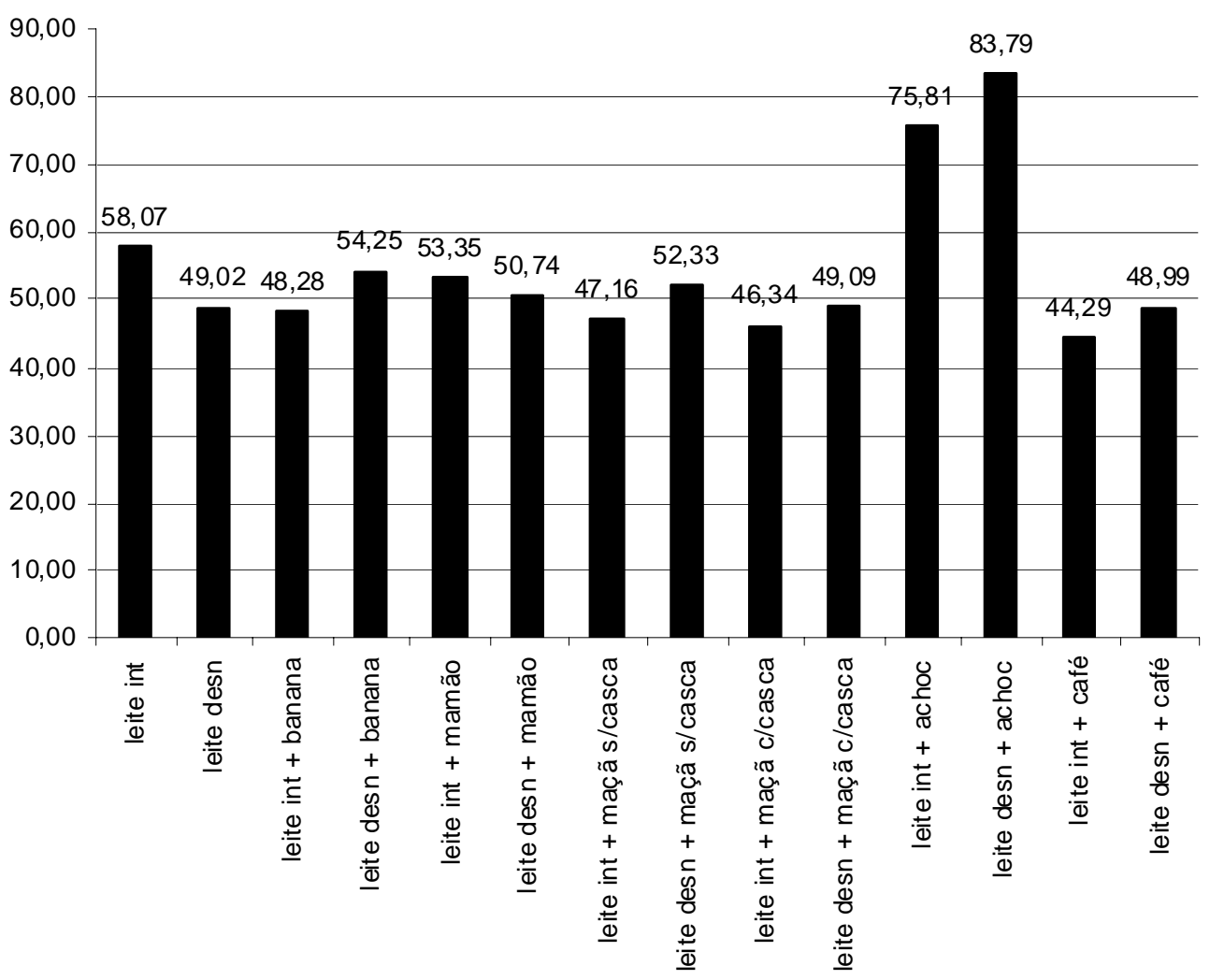

Considerando a recomendação diária de cálcio preconizada pela resolução RDC $n^{\circ} 269$, de 22 de setembro de 2005 (BRASIL, 2005), e as quantidades de cálcio encontradas nas amostras podem ser observadas as porcentagens de cálcio que são fornecidas pelas misturas. A porção de $200 \mathrm{~mL}$ (em média um copo) de leite integral adicionado de achocolatado em pó fornece $30,33 \%$ da ingestão diária recomendada (IDR) de cálcio para crianças de 1 a 3 anos de idade; 25,27\% da IDR para crianças de 4 a 6 anos de idade; $21,66 \%$ da IDR para crianças de 7 a 10 anos de idade; 12,63\% da IDR para gestantes e $15,16 \%$ da IDR para lactantes e adultos. Considerando ainda a mesma resolução e a mesma porção, a amostra de leite desnatado adicionado de achocolatado em pó fornece 33,52\%; 27,93\%; 23,94\%; 13,96\% e 16,76\% da IDR para crianças de 1 a 3 anos; crianças de 4 a 6 anos; crianças de 7 a 10 anos; gestantes e adultos e lactantes, respectivamente.

\section{CONCLUSÃO}

De acordo com os resultados obtidos foi constatada apenas correlação significativa positiva do ferro em relação à disponibilidade de cálcio. Os demais componentes analisados não apresentaram correlação significativa. 
Em termos de quantidade de cálcio dialisado, as amostras compostas somente por leite integral e leite desnatado e, as amostras formadas por misturas de leite (integral e desnatado) com achocolatado mostraram os maiores teores.

Com relação à disponibilidade de cálcio, as amostras compostas por misturas com banana e mamão apresentaram os melhores resultados.

As amostras compostas por misturas de leite com maçã sem casca apresentaram resultados para a disponibilidade de cálcio ligeiramente superiores que as amostras formadas por misturas com maçã com casca. Em termos de cálcio dialisado não foi verificada interferência da presença da casca na mistura.

As amostras compostas por misturas de leite integral e leite desnatado com achocolatado em pó apresentaram maiores quantidades de cálcio disponível por porção de $200 \mathrm{~mL}$.

\section{ABSRACT}

\section{CALCIUM AVAILABILITY IN WHOLE AND SKIMMED MILK ADDED OF FRUITS, COFFEE AND CHOCOLATE POWDER}

The aim of this study was to evaluate the calcium availability in whole and skimmed cow milk (UHT) mixed with banana (Nanica cultivar); papaya (Formosa cultivar); apple (Gala cultivar) with and without peel; chocolate powder and coffee infusion. The samples were submitted to analysis of centesimal composition, antinutritional factors (tannins, oxalic acid and phytic acid), minerals and calcium availability. Randomized complete block design was adopted and the results were submitted to statistical analysis using the Tukey test, and the correlation Pearson test in the level of 5\%. As for the centesimal and mineral composition and the available calcium, only the lipid component presented significant difference among the samples composed by whole milk and the composed by skimmed milk. Only the iron presented significant positive correlation to the calcium availability. The samples composed by whole and skimmed milk, both mixed with banana and papaya presented the best results to the calcium availability. However the samples made from the mixture of whole and skimmed milk with chocolate powder presented numerical results higher to the other analyzed samples, in relation to the quantity of calcium, dialyzed calcium available in portions of $200 \mathrm{~mL} / \mathrm{sample}$, probably due to the chocolate powder formula. Taking in consideration the obtained data it can be concluded that the best results were obtained with whole and skimmed milk with chocolate powder and banana. The apple peel did not influence in the result of the calcium availability.

KEY-WORDS: MILK; FRUIT; CHOCOLATE POWDER; COFFEE; CALCIUM - AVAILABILITY.

\section{REFERÊNCIAS}

1 ÁNGEL, M.; MENDONZA, R.; MIGUEL, J.; PULIDO, E.; ZÚNIGA, R.M.; CLEOFAS, M.; ARRIOLA, R. Osteoporosis em mexicanas mayores de 40 años. Revista Medica del Instituto Mexicano del Seguro Social, v.41, n.3, p.193-202, 2003.

2 AQUINO, R. C.de; PHILIPPI, S.T. Consumo infantil de alimentos industrializados e renda familiar na cidade de São Paulo. Revista de Saúde Pública, v.36, n.6, p.655-660, 2002.

3 ABPM. Associação Brasileira dos Produtores de Maçã. Maçã-propriedades. Disponível em: http:// www.abpm.org.br. Acesso em: 27 jan. 2006.

4 AOAC. Association of Official Analytical Chemists. Official methods of the AOAC International. $20^{\text {th }}$ ed. Washington, 2006. $1 \mathrm{v}$.

5 BLIGH, E.G.; DYER, W.J. A rapid method of total lipid extraction and purification. Canadian Journal of Biochemistry and Physiology, v.37, n.8, p.911-917, 1959.

6 BRASIL. Ministério da Agricultura, Pecuária e Abastecimento. Resolução n. 2, de 19 de novembro de 2002. Disponível em: http://www.agricultura.gov.br/. Acesso em: 20 jun. 2007.

7 Resolução RDC n. 360, de 23 de dezembro de 2003. Disponível em: http://www.anvisa.gov.br/ e-legis/. Acesso em: 20 jun. 2007. 

de setembro de 2005. Disponível em: http://www.anvisa.gov.br/e-legis/. Acesso em: 10 jan. 2007.

9 BOAVENTURA, A.C. Avaliação química, protéica e biodisponibilidade de cálcio nas folhas de couvemanteiga, couve-flor e espinafre. Piracicaba, 1998. 46 f. Dissertação (Mestrado), Escola Superior de Agricultura "Luiz de Queiroz", Universidade de São Paulo.

10 BOSSCHER, D.; DYCK, K.V.; ROBBERECHT, H.; VAN CAILLIE-BERTRAND, M.; DEELSTRA, H. Bioavailability of calcium and zinc from cow's milk-based versus soya-based infant food. International Journal of Food Science and Nutrition, v.49, n.4, p.277-283, 1998.

11 BOSSCHER, D.; VAN CAUWENBERG, R.; VAN DER AUWERA, J.C.; ROBBERECHT, H.; DEELSTRA, H. Calcium, iron and zinc availability from weaning meals. Acta Paediatrica, v.91, p.761-768, 2002. Disponível em: http//www.journalsonline.tandf.co.uk/content/a467gel4v6mhpce8/fulltext. Acesso em: 28 nov. 2006.

12 BOSSCHER, D.; VAN CAILLIE-BERTRAND, M.; DEELSTRA, H. Effect of thickening agents, based on soluble dietary fiber, on the availability of calcium, iron, and zinc from infant formulas. Nutrition, v.17, n.7/ 8, p.614-618, 2001. Disponível em: http//www.sciencedirect.com/science?_ob. Acesso em: 28 nov. 2006.

13 CARMO, M.B.do; TORAL, N.; SILVA, M.V.da; SLATER, B. Consumo de doces, refrigerantes e bebidas com adição de açúcar entre adolescentes da rede pública de ensino de Piracicaba, São Paulo. Revista Brasileira de Epidemiologia, v.9, n.1, p.121-130, 2006.

14 CRAWFORD, P.B.; WANG, M.C.; SABRY, Z.I.; HUDES, M.; VanLOAN, M.; BACHARACH, L.K. Adolescent diet in predictive of peak bone mass. American Journal of Clinical Nutrition, v.75, n.2, p.356, 2002. Disponível em: http://www.ajcm.org. Acesso em: 8 jan. 2007. Suppl.

15 CURTI, F. Efeito da maçã gala (Malus domestica Bork) na lipidemia de ratos hipercolesterolêmicos. Piracicaba, 2003. 75 f. Dissertação (Mestrado), Escola Superior de Agricultura "Luiz de Queiroz", Universidade de São Paulo.

16 EPAGRI. Empresa de Pesquisa Agropecuária e Extensão Rural de Santa Catarina. A Cultura da macieira. Florianópolis, 2002. 743 p.

17 ERVITI, J. Utilización de fármacos para la osteoporosis. Anales del Sistema Sanitário de Navarra, v.26, Suppl. 3, p.107-121, 2003.

18 FARIAS JÚNIOR, G.de; OSÓRIO, M.M. Padrão alimentar de crianças menores de cinco anos. Revista de Nutrição, v.18, n.6, p.793-802, 2005.

19 FAO. Food and Agriculture Organization. Production of fruits and vegetables and share in world 2004. Disponível em: http://www.fao.org/es/ess/yearbook/vol_1_1/pdf/b03.pdf. Acesso em: 04 abr. 2007.

20 FRANCO, G. Tabela de composição química dos alimentos. 9.ed. São Paulo: Atheneu, 2001. 307 p.

21 FRAZÃO, P.; NAVEIRA, M. Prevalência de osteoporose: uma revisão crítica. Revista Brasileira de Epidemiologia, v.9, n.2, p.206-214, 2006.

22 GRYNSPAN, F.; CHERYAN, M. Phytate-calcium interactions with soy protein. Journal of the American Oil Chemists Society, v.66, n.1, p.93-96, 1989.

23 GUÉGUEM, L.; POINTILLART, A. The bioavailability of dietary calcium. Journal of the American College of Nutrition, v.19, n.2, p.119-136, 2000

24 GUIMARÃES, L.R.; BRAUM, M.L.; GOMEZ, R. Medidas terapêuticas na prevenção e tratamento da osteoporose. Nutrição em Pauta, v.13, n.73, p.28-33, 2005.

25 HEANEY, R.P.; WEAVER, C.M.; FITZSIMMONS, M.L. Soybean phytate content: effect on calcium absorption. American Journal of Clinical Nutrition, v.53, n.3/4, p.745-747, 1991.

26 ITO, M.S.B. Tabela de composição de alimentos - USP: banco de dados de alimentos industrializados. São Paulo, 2003. 206 f. Dissertação (Mestrado), Faculdade de Ciências Farmacêuticas, Universidade de São Paulo. 
27 KENNEFICK, S.; CASHMAN, K.D. Investigation of an in vitro model for predicting the effect of food components on calcium availability from meals. International Journal of Food Sciences and Nutrition, v.51, p.45-54, 2000.

28 KOVALESKY, A. Maçã: fitossanidade. Bento Gonçalves: Embrapa Uva e Vinho; Brasília: Embrapa Informação Tecnológica, 2004. 85 p. (Frutas do Brasil, 38).

29 KOWALSKI, S.C.; SJENZFELD, V.L.; FERRAZ, M.B. Utilização de recursos e custos em osteoporose. Revista da Associação Médica Brasileira, v.47, n.4, p.352-357, 2001.

30 LERNER, R.B.; LEI, D.L.M.; CHAVES, S.P.; FREIRE, R.D. O cálcio consumido por adolescentes de escolas públicas de Osasco, São Paulo. Revista de Nutrição, v.13, n.1, p.57-63, 2000.

31 MAESTRO, V. Padrão alimentar e estado nutricional: caracterização de escolares de municípios paulista. Piracicaba, 2003. 130 f. Dissertação (Mestrado), Escola Superior de Agricultura "Luiz de Queiroz", Universidade de São Paulo.

32 MATKOVIC, V. Calcium intake and peak bone mass. New England Journal of Medicine, v.327, n.2, p.119-120, 1992.

33 McCLUNG, M.R. Prevention and management of osteoporosis. Best Practice \& Research Clinical Endocrinology \& Metabolism, v.17, n.1, p.53-71, 2003.

34 MELLO, L.M.R.; BORGES JÚNIOR, L. Mercado nacional e internacional. In: KOVALESKY, A. Maçã: póscolheita. Bento Gonçalves: Embrapa Uva e Vinho; Brasília: Embrapa Informação Tecnológica, 2004. $109 \mathrm{p}$.

35 MICROSOFT. Microsoft Office xp. São Paulo, 2003.

36 MOIR, K.W. The determination of oxalic acid in plants. Queensland Journal of Agriculture Science, v.10, p.1-3, 1953.

37 OMS. Organização Mundial da Saúde. Dieta, nutrición y prevención de enfermedades crónicas. Genebra, 2003. 181 p. (Série de Informes Técnicos, 916).

38 PHILIPPI, S.T. Tabela de composição de alimentos: suporte para decisão nutricional. Brasília: ANVISA, FINATEC/NUT - UnB, 2001. 107 p.

39 PRICE, P.W.; BOUTON, E.E.; GROSS, P.; Mac THERON, B.A.; THOMPSON,J.N.; WEISS, A.E. Interactions among three thophic levels: influence of plants on interactions between insect herbivores and natural enemies. Annual Review of Ecology and Sytstematics, v.11, p.41-65, 1980.

40 ROIG, M.J.; ALEGRIA, A.; BARBERÁ, R.; FARRÉ, R.A.; LAGARDA, M.J. Calcium bioavailability in human milk, cow milk and infant formulas-comparison between dialysis and solubility methods. Journal of Food Chemistry, v.65, p.353-357, 1999a.

41 Calcium dialysability as an estimation of bioavailability in human milk, cow milk and infant formulas. Journal of Food Chemistry, v.64, p. 403-409, 1999b.

42 SANTOS, J.S.; COSTA, M.C.O; NASCIMENTO SOBRINHO, C.L.; SILVA,M.da C.M.da; SOUZA, K.E.P.de; MELO, B.O. Perfil antropométrico e consumo alimentar de adolescentes de Teixeira Freitas - Bahia. Revista de Nutrição, v.18, n.5, p.623-632, 2005.

43 SARRUGE, J.R.; HAAG, H.P. Análises química em plantas. Piracicaba: ESALQ, Depto. Química, 1974. 50 p.

44 SILVA, C.C. da; TEIXEIRA, A.S.; GOLDBERG, T.B.L. Impacto da ingestão de cálcio sobre a mineralização óssea em adolescentes. Revista de Nutrição, v.17, n.3, p.351-369, 2004.

45 SAS INSTITUTE. Statistical Analysis System. Version 6. Cary, 1996. 1 CD-ROM.

46 TAHVONEN, R. Contents of selected elements in some fruits, berries, and vegetables on the finish market in 1987-1989. Journal of Food Composition and Analysis, v.6, p.75-86, 1993.

47 ÜNAL, G.; NEHIR, S. El; KILIÇ, S. In vitro determination of calcium bioavailability of milk, dairy products and infant formulas. International Journal of Food Sciences and Nutrition, v.56, n.1, p.13-22, 2005. 
48 UNICAMP. Universidade de Campinas. Tabela brasileira de composição de alimentos - TACO. 2.ed. Campinas, 2006. Disponível em: http://www.unicamp.br/nepa/taco/contar/taco_versao2.pdf. Acesso em: 19 abr. 2007.

49 USP. Universidade de São Paulo. Tabela brasileira de composição de alimentos. São Paulo, 2004. versão 4.1. Disponível em: http://www.fcf.usp.br/tabela/. Acesso em: 19 abr. 2007. 